\title{
Coverage and Deployment Analysis of Narrowband Internet of Things in the Wild
}

\author{
Konstantinos Kousias, Giuseppe Caso, Özgü Alay, Anna Brunstrom, Luca De Nardis, \\ Maria-Gabriella Di Benedetto and Marco Neri
}

\begin{abstract}
Narrowband Internet of Things (NB-IoT) is gaining momentum as a promising technology for massive Machine Type Communication (mMTC). Given that its deployment is rapidly progressing worldwide, measurement campaigns and performance analyses are needed to better understand the system and move toward its enhancement. With this aim, this paper presents a large scale measurement campaign and empirical analysis of NB-IoT on operational networks, and discloses valuable insights in terms of deployment strategies and radio coverage performance. The reported results also serve as examples showing the potential usage of the collected dataset, which we make opensource along with a lightweight data visualization platform.
\end{abstract}

\section{INTRODUCTION}

As part of beyond-4G and 5G systems, the machine type communication (MTC) paradigm provides the ideal substrate toward cellular internet of things (IoT), and is leading to a shift in cellular network design and deployment. On the one hand, MTC introduces a novel degree of heterogeneity, given that the things, i.e., autonomous devices with novel features and requirements, must be integrated into the mobile network, which was originally designed for serving humans with their peculiar traffic. On the other hand, it requires long term performance analyses for stable network deployment, in particular when its massive nature (mMTC), in terms of the unprecedented number of devices, is considered [1] [2].

A relevant step toward enabling mMTC is represented by the 2016 Release 13 (Rel-13) standard by the 3rd generation partnership project (3GPP), where three technologies were proposed: Extended Coverage Global System for Mobile Communications (GSM) IoT (EC-GSM-IoT), Long Term Evolution (LTE) for MTC (LTE-M), and Narrowband IoT (NB-IoT) [2]. These represent the cellular options for so-called low power wide area networks (LPWANs), which aim to deliver massive IoT services over wide areas, i.e., several kilometers, with low costs and power consumption [3].

Since Rel-13, and considering the advances in Rel-14 (2017) and Rel-15 (2018), NB-IoT is triggering significant attention across researchers and operators as one of the most appealing LPWANs [4] [5] [6]. Hence, the theoretical aspects of NB-IoT are being formalized and analyzed, while several mobile operators are launching and making operational NB-IoT networks worldwide [7].

K. Kousias is with Simula Research Laboratory.

G. Caso is with Simula Metropolitan CDE.

Ö. Alay is with University of Oslo and Simula Metropolitan CDE.

A. Brunstrom is with Karlstad University.

L. De Nardis and M.-G. Di Benedetto are with Sapienza Uni. of Rome.

M. Neri is with Rohde\&Schwarz.
As the NB-IoT deployment is progressing at a rapid pace, field trials and measurement campaigns become of extreme interest, considering that these are the very first attempts of enabling IoT services on the cellular architecture, and thus a closer empirical look is needed to better understand and optimize the system. To this end, data-driven analyses are crucial for both researchers and operators, as they allow to identify correlations and causalities between deployment choices and performance, highlight encountered challenges, and derive guidelines for further research and development. However, extensive measurement campaigns are often scarcely available to researchers, and rather costly and time consuming for operators, which may thus opt for less expensive but suboptimal alternatives. These include simulation-based studies [8], which provide general analyses that cannot perfectly match with real scenarios.

Considering the above motivations, this paper presents a large scale measurement campaign of NB-IoT coverage for two Norwegian and three Italian operators, conducted in the cities of Oslo and Rome during 2019. To the best of our knowledge, this represents the first empirical analysis of NB-IoT performance on operational networks, which considers coverage aspects across heterogeneous scenarios and environments. The main contributions of this paper are:

- We conduct an analysis of the strategies being adopted for deploying NB-IoT, aiming to highlight implementation trends and derive takeaways for improvement.

- We assess NB-IoT coverage, depicting how the current system deployment reflects in service availability across urban scenarios.

- We conduct a comparison between two NB-IoT spectrum operation modes, in-band and guard-band, to understand NB-IoT's coexistence with LTE.

- We open-source our dataset, comprising of NB-IoT and LTE coverage measurements in Oslo and Rome, to support the discovery of new insights and research. We also provide a web platform for geo-referenced visualization of the collected data [9].

The article is organized as follows: a brief description of NB-IoT technology is first provided, followed by an overview of the experimental design. We then discuss our findings and conclude our work.

\section{TECHNOLOGY Description}

NB-IoT is a radio interface implemented over the cellular licensed spectrum. It offers high deployment flexibility and 
integration with the existing architecture, minimizing costs and complexity at network and device sides, and providing performance in line with mMTC expectations. Below, we describe NB-IoT operation modes, possible deployment strategies, and coverage aspects, which are the focus of this paper. Moreover, we mention the main features in Rel-13 [4], since this release is being mostly deployed, as confirmed by our measurement campaign. We refer the reader to [2] [10] for the analysis of NB-IoT advances in Rel-14 and Rel-15.

Operation modes and deployment strategies: NB-IoT devices operate over either a $200 \mathrm{kHz}$ GSM-like channel or an LTE physical resource block (PRB) of $180 \mathrm{kHz}$, allowing coexistence with both GSM and LTE. Three different operation modes are defined:

- stand-alone, which uses a $200 \mathrm{kHz}$ channel obtained by refarming the GSM spectrum,

- in-band, which uses a single PRB within a set of PRBs used by LTE, selected in order to minimize interference from/to LTE, and

- guard-band, which leverages a PRB within a guard band among different sets of LTE PRBs.

After selecting one of the modes, the operators can provide NB-IoT services via a software upgrade of their infrastructure, i.e., enhancing the capabilities of their E-UTRAN Node Bs (eNBs) and cells, at least in areas where these are already present for serving broadband users.

The operators can select several deployment strategies and differently leverage the trade-off between costs and performance. In particular, given a broadband area, e.g., an urban environment, operators may either activate an NB-IoT carrier in all already deployed LTE eNBs and cells, or select some of them. The first option is somehow simpler, since it does not require in-depth analysis for coverage optimization. However, it may increase the operational costs, including energy consumption, and result in redundant deployment when NB-IoT use cases and coverage enhancement (CE) techniques are considered (see later in this section). The second option requires a more careful planning, but may lead to a better trade-off between costs and quality of service (QoS).

Specifically for the in-band mode, the coexistence with LTE is another aspect to consider when deciding between the two aforementioned options. On the one hand, NB-IoT activation in specific eNBs/cells leads to possible interference from/to LTE, since the LTE-only eNBs/cells may use the PRB dedicated to NB-IoT for their traffic. On the other hand, NB-IoT full deployment leads to sub-optimal resource usage, since a specific PRB is exclusively dedicated to infrequent and sporadic NB-IoT traffic, at the expenses of LTE endusers. Moreover, in areas with low-to-null broadband coverage, e.g., rural and deep indoor environments, the operators may apriori install dedicated but costly eNBs, or first check whether NB-IoT CE techniques allow the reuse of the existing infrastructure, triggering the installation only in cases of negative response.

CE techniques: NB-IoT targets service reliability and delaytolerant uplink (UL) data exchange. Hence, advanced modulation and coding schemes are not supported. Rather, CE techniques are used, aiming to favour connectivity in harsh environments, such as dense urban and deep indoor. A first $\mathrm{CE}$ effect is obtained by narrowing down the bandwidth with respect to LTE, since this focuses the transmitted power on smaller bands, at the cost of reducing the data rate. Moreover, NB-IoT standards allow repeated transmissions, which increase the probability of correct reception. In particular, downlink (DL) and UL signals can be repeated up to 2048 and 128 times, respectively. The number of repetitions depends on radio conditions and operator configurations, these latter transmitted in master information block (MIB) and system information block (SIB) messages. Repetition settings are given in SIB2 messages.

The device estimates its coverage conditions during the random access (RA) procedure, which triggers the connection to a cell, i.e., the one detected with highest reference signal received power (RSRP) $[\mathrm{dBm}]$. The comparison between RSRP and operator-defined thresholds allows to estimate a coverage level (CL) [11]. Up to two thresholds can be defined, leading to three possible CLs; CLO represents LTE-like radio conditions, while CL1 and CL2 apply to challenging scenarios requiring more repetitions. During consecutive RA attempts the device can adjust its CL estimate and move to higher CLs, if it experiences connection failures in the first attempts.

Other Features: DL and UL resources are accessed in Frequency Division Duplex (FDD) mode. Orthogonal Frequency Division Multiple Access (OFDMA) is applied in DL, with $15 \mathrm{kHz}$ subcarrier spacing. The PRB is divided into seven OFDM symbols of twelve subcarriers each, and occupies $0.5 \mathrm{~ms}$. Single Carrier Frequency Division Multiple Access (SC-FDMA) is applied in UL, with a subcarrier spacing of 15 $\mathrm{kHz}$ or $3.75 \mathrm{kHz}$.

NB-IoT devices can be idle and connected. Idle devices are not functionally connected to the network, and actuate the procedures for switching into connected, including cell selection and tracking of control messages (paging monitoring). After selecting a cell, the devices transit from idle to connected via RA, and exchange data while continuing paging monitoring.

Targeting energy efficiency and long device battery lifetime, NB-IoT standards introduce (i) extended discontinuous reception $(e D R X)$, which allows to perform paging monitoring more infrequently compared to LTE, and (ii) power saving mode (PSM), which allows idle devices to disconnect the radio and minimize energy consumption [12].

Comparison with other LPWANs: NB-IoT plays a leading role across LPWANs, with constantly increasing market shares [3] [13]. Compared to other 3GPP technologies, NB-IoT shares some features and use cases with EC-GSM-IoT, but provides lower device complexity and better integration with GSM, LTE, and 5G. NB-IoT and LTE-M target complementary applications, with LTE-M having higher device complexity and costs. Considering LPWANs in the unlicensed spectrum, NB-IoT has a competitor in Long Range Wide Area Network (LoRaWAN). They provide comparable throughput and latency performance, and adopt different security mechanisms. NB-IoT outperforms LoRaWAN in terms of communication reliability, due to the use of licensed spectrum and wellestablished infrastructures [2]. 


\section{EXPERIMENTAL DESIGN}

In the above overview, we highlight NB-IoT deployment and coverage aspects discussed in this paper. We now present our measurement campaigns and analyses. Particularly, in this section we describe the adopted hardware and software components, and summarize experimental setup and collected dataset. Finally, we introduce our data visualization framework.

\section{A. Measurement System}

For the NB-IoT measurements in Oslo and Rome, we used the Rohde\&Schwarz (R\&S) TSMA6 toolkit, an Exelonix Narrowband (NB) USB device, and a global positioning system (GPS) antenna. TSMA6 is a system integrating:

- A spectrum scanner, for passive measurement of 3GPP technologies up to $6 \mathrm{GHz}$, including $5 \mathrm{G}$ New Radio (NR). It supports NB-IoT signal detection and decoding in inband, guard-band, and stand-alone.

- A laptop, where the controlling software, named ROMES4, is installed. Combined with scanner and device, i.e., the Exelonix module in our case, and exploiting GPS georeference, ROMES4 provides an overview of coverage, interference, and QoS measurements.

We leveraged two further features from TSMA6, i.e., the automatic channel detection, which performs automatic detection of active channels for all technologies in the specified spectrum, and the base transceiver station position estimation, which combines passive measurements and GPS to estimate the position of the cells forming the operators' infrastructures.

The Exelonix module is a Qualcomm-based device supporting NB-IoT and LTE-M. We embedded the module with NB-IoT SIM cards from the operators under test, and used it to monitor the radio conditions of the serving cell, and execute repeated connections to the operators' networks. Hence, we are able to analyze the RA procedure, including the CL estimation, aiming to reveal further aspects related to operator-specific configurations, e.g., how the RSRP thresholds adopted in the CL estimation impact the perceived coverage.

\section{B. Measurement Campaigns}

We performed two measurement campaigns. The first campaign was designed to explore city-wide coverage and deployment aspects under heterogeneous scenarios, and covered a period of three weeks during summer 2019 in Oslo, Norway. We enabled the scanner to perform passive measurements on four LTE bands (including guard bands), i.e., Band 1, 3, 7, and 20, and detected three LTE operators, denoted in the following as $\mathrm{Op}_{\mathrm{k}, \mathrm{N}}$, where $\mathrm{k}$ identifies the operator and $\mathrm{N}$ stands for Norway. To guarantee reliability and completeness, we conducted measurements in various city areas and different scenarios, that we label as deep indoor (DI) (14), for basements and deep enclosed spaces, indoor (I) (48), for houses and multifloor buildings, outdoor walking $(O W)(8)$, for outdoor while walking, and outdoor driving (OD) (14), for outdoor while on public transport. Numbers in parenthesis represent the number of sub-campaigns for each scenario. We replicated a subset of our measurements over time (i.e., morning vs. afternoon vs. evening, and week vs. weekend), to account for temporal effects.

In the second campaign we collected a smaller dataset composed of 3 sub-campaigns (one for $I$ and two for $O D$ scenarios), within a couple of days of 2019 in Rome, Italy, to compare the performance between in-band and guard-band modes. The dataset features measurements related to three operators $\left(O \mathrm{p}_{\mathrm{k}, \mathrm{I}}\right.$, where $\mathrm{k}$ identifies the operator and I stands for Italy) in Band 20. At the time of the collection, $\mathrm{Op}_{2, \mathrm{I}}$ and $\mathrm{Op}_{3, \mathrm{I}}$ were deploying NB-IoT in guard-band, while $\mathrm{Op}_{1, \mathrm{I}}$ was testing the in-band option. This makes the dataset fitting for a comparison between the two modes. We report that, as confirmed by following tests, $O \mathrm{p}_{1, \mathrm{I}}$ has moved toward a guard-band deployment. However, the dataset remains valid for comparing the two modes.

The complete dataset consists of 1.2M LTE and $1.4 \mathrm{M}$ NB-IoT passive scans for Oslo, and $121 \mathrm{~K}$ LTE and $51 \mathrm{~K}$ NB-IoT passive scans for Rome. The list of collected attributes is provided in [9]. To anonymize the operators' identity, mobile network code (MNC), E-UTRA absolute radio frequency channel number (EARFCN), and cell ID (CID) are given as references and not associated to real values.

\section{Visualization}

Designing and implementing a platform that enables interactive geo-spatial visualization is beneficial for discovering operators' eNBs spatial deployment and pinpointing at a glance areas with limited coverage. Thereby, we design an open-source visualization platform showing eNB placement and coverage for each operator under test [9]. We implement the platform using an $R$ interface to Leaflet, an open-source JavaScript library for mobile-friendly maps. Users can exploit several features, from controlling which layers they see on the map to dynamically altering the observed coverage based on the zoom level. We include additional plugins and add-ons to enhance end-user experience.

\section{Performance Evaluation}

The deployment of the cellular radio access network (RAN) is driven by the need of optimizing the coverage and making the service accessible to end-users. This aspect is more challenging for cellular IoT technologies, as they are expected to mostly leverage the existing infrastructure, which is however tailored for broadband services. In this section, we present the results of our measurement analysis, which contrast deployment strategies and coverage performance for the two operators currently providing NB-IoT in Oslo. We also study the implications of deploying NB-IoT in in-band or guardband, by leveraging the dataset collected in Rome.

\section{A. Network Deployment Strategy}

RAN deployment is a challenging optimization task [14]. The targets of the operators include (i) to ensure sufficient coverage, (ii) to satisfy QoS requirements, and (iii) to efficiently deal with energy and cost constraints. Hence, they aim 


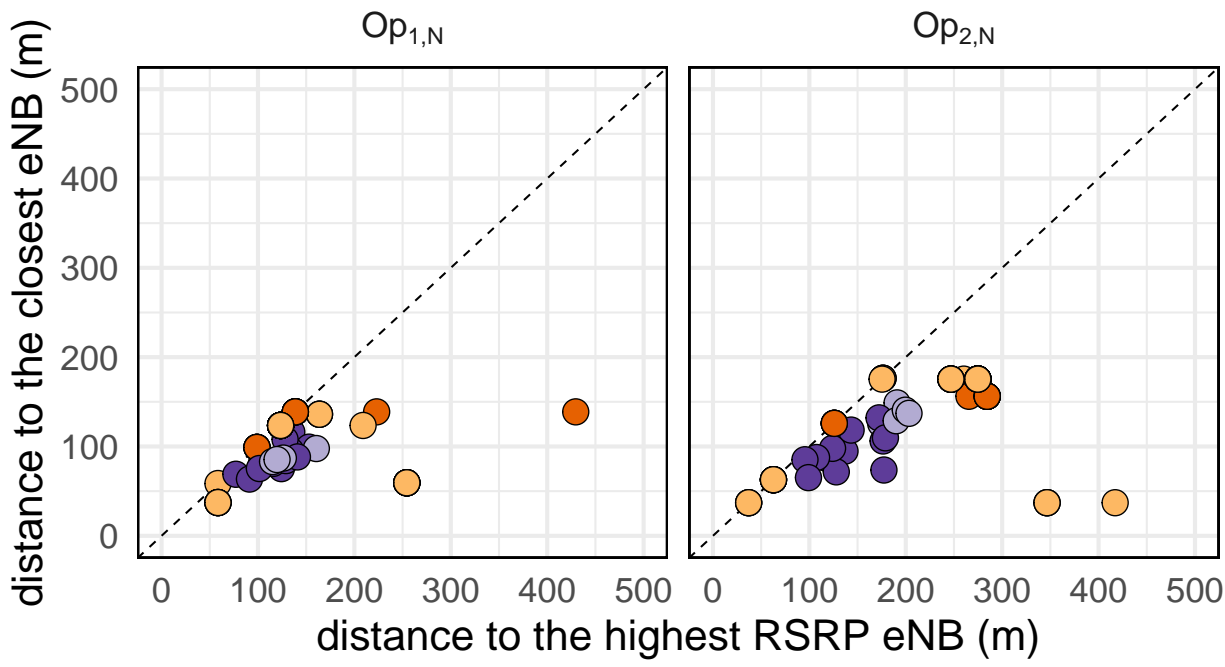

$\bigcirc$ DI $\bigcirc$ I $\bigcirc$ OW $\bigcirc$ OD

Fig. 1: Scatterplot between average distance to the eNB with highest RSRP and average distance to the nearest eNB for Op $p_{1, N}$ (left) and $\mathrm{Op}_{2, \mathrm{~N}}$ (right). Different colors represent different scenarios.

to optimize the eNB placement by considering environmental characteristics, i.e., density and structure of surrounding buildings. However, business expenses, radiation safety levels, and interference between cells, are some aspects that limit the qualified spots for installing an eNB, hence favoring alternative locations. Next, we empirically analyze the deployment strategies for operators providing NB-IoT in Oslo.

Deployment statistics: Table I provides per-operator statistical insights with respect to the number of detected eNBs and EARFCNs used for NB-IoT and LTE. We observe that, across the monitored bands, $\mathrm{Op}_{1, \mathrm{~N}}$ and $\mathrm{Op}_{2, \mathrm{~N}}$ have activated one NB-IoT carrier each in the guard bands of Band 20. Considering the infrastructure, $\mathrm{Op}_{1, \mathrm{~N}}$ is dominant in terms of number of eNBs for both technologies, implying a denser deployment compared to $\mathrm{Op}_{2, \mathrm{~N}}$. Both operators leverage the existing LTE infrastructure for deploying NB-IoT, with no additional eNBs installed. In this regard, nearly $86 \%$ of the detected LTE eNBs have been reconfigured for NB-IoT. The few NB-IoT-only eNBs detected for $\mathrm{Op}_{1, \mathrm{~N}}$ could be explained by considering the more penetrating nature of NB-IoT, or should be appointed to other causes that prevented LTE detection. We also observe that the eNBs not supporting NB-IoT are in $90 \%$ of the cases operating at a band other than Band 20. This indicates that almost all eNBs operating in Band 20 support NB-IoT. Finally, we highlight that the operators leverage a different number of EARFCNs for LTE, while only one for NB-IoT.

Deployment optimality: RSRP is a key metric for handover and cell (re-)selection phases, hence, it is a critical parameter when evaluating how radio coverage is affected by the network infrastructure. In the following, we evaluate deployment optimality, which assumes given a location, the closest eNB would offer the highest RSRP under ideal propagation and environmental scenarios, and also assuming constraintless eNB placement. In practice though, several factors may inhibit this
TABLE I: Network deployment statistics with regard to number of eNBs and EARFCNs per technology. eNBs\% is defined as the ratio between the number of LTE/NB-IoT eNBs and the total number of eNBs. Absolute numbers are provided in parenthesis.

\begin{tabular}{|c|c|c||c|c|}
\hline & \multicolumn{2}{|c||}{ eNBs\% } & \multicolumn{2}{c|}{ EARFCNs } \\
\hline & LTE & NB-IoT & LTE & NB-IoT \\
\hline $\mathrm{Op}_{1, \mathrm{~N}}$ & $96.6 \%(167)$ & $84.3 \%(146)$ & 6 & 1 \\
$\mathrm{Op}_{2, \mathrm{~N}}$ & $100 \%(122)$ & $87.7 \%(107)$ & 4 & 1 \\
$\mathrm{Op}_{3, \mathrm{~N}}$ & $100 \%(70)$ & NA & 2 & NA \\
\hline
\end{tabular}

situation, particularly in dense urban environments, such as multipath propagation, network congestion and interference, and constrained eNB placement.

Figure 1 shows whether the operators are close to an optimal NB-IoT deployment in Oslo. For each location in a sub-campaign, we compute (i) the distance toward the eNB detected with highest RSRP, and (ii) the distance toward the nearest eNB, both in meters. Then, for each sub-campaign, we average across all locations. In an optimal deployment situation, we expect a linear relationship between the two distances. Thereby, the deviation from the diagonal represents how far the deployment refrains from being optimal. We observe that both operators approach deployment optimality in several indoor scenarios, while slightly deviate in outdoor sub-campaigns. In particular, $\mathrm{Op}_{1, \mathrm{~N}}$ mostly works in a short distance regime, i.e., less than 150 meters, due to its dense infrastructure. $O p_{2, N}$ deviates from the identity line more frequently, and several sub-campaigns present distances exceeding 150 meters, thus hinting sub-optimal deployment. For both operators, a negative joint impact of propagation conditions and deployment suboptimality is highlighted by large deviations observed for specific DI and I sub-campaigns.

Takeaways: In initial deployment phases, the operators are actuating rather dense NB-IoT deployment strategies, with large amounts of pre-existing eNBs now supporting NB-IoT. 


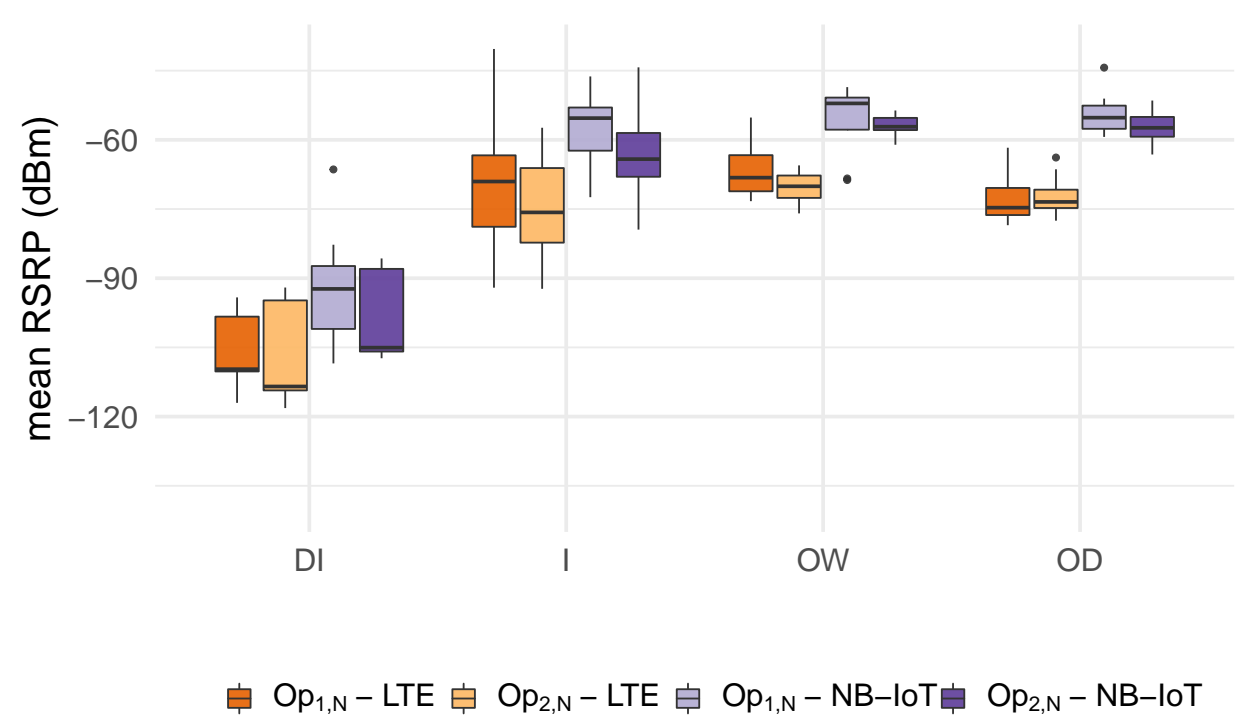

Fig. 2: Sub-campaign average coverage in terms of RSRP $[\mathrm{dBm}]$, grouped by scenario and divided by a combination of operator $\left(\mathrm{Op}_{1, \mathrm{~N}}\right.$, $\mathrm{Op}_{2, \mathrm{~N}}$ ) and technology (LTE, NB-IoT).

This solution is sub-optimal in terms of operational costs, and leads to increased carbon emissions, for which the RAN is already the main contributor across network functions [13]. The analysis of real deployments can support the derivation of optimization strategies, e.g., dynamic (de-)activation of specific eNBs, moving toward green cellular IoT.

\section{B. Radio Coverage}

We now analyze NB-IoT coverage for both operators, showing how it changes across scenarios, and exploiting the LTE dataset for comparison. As above, due to its importance in cellular systems, we consider RSRP as a key indicator. In particular, for each measurement location in a sub-campaign, the coverage for an operator is defined as the highest RSRP perceived among all the CIDs detected for that operator. We then express the sub-campaign average coverage, by averaging the RSRP across locations.

Technology and scenario comparison: Figure 2 depicts the distribution of average RSRP in a boxplot format with sub-campaigns grouped per scenario and colored by operator and technology. We validated the statistical significance by leveraging the Kruskal-Wallis and Dunn's tests, aiming to identify which distributions have statistically different mean values. Due to space limitations, we report the results in [9].

We observe that, compared to LTE, NB-IoT provides statistically significant RSRP boosts of 11.73, 12.29, 12.06 and $16.71 \mathrm{~dB}$ on average for each scenario, respectively. This result is in line with the power boosting expected by 3GPP TS 36.104 [15], which is at least $+6 \mathrm{~dB}$ when evaluated as the difference between the power of the entire NB-IoT carrier $(180 \mathrm{kHz})$ and the average power over all carriers (LTE and NB-IoT).

We also compare NB-IoT average RSRP across scenarios. In particular, a statistically significant increase of 36.36 and $35.70 \mathrm{~dB}$ for $\mathrm{Op}_{1, \mathrm{~N}}$ and $\mathrm{Op}_{2, \mathrm{~N}}$, respectively, is observed when comparing I with DI scenarios. This shows the negative effect
TABLE II: Ratio of being in a specific CL, grouped by scenario, for $O p_{1, N}$ and $O p_{2, N}$. The ratio is evaluated as the number of readings for a CL divided by the number of readings for all CLs.

\begin{tabular}{|c|c|c|c|c|c|c|c|c|c|c|c|c|}
\hline Operators & \multicolumn{12}{|c|}{ Scenarios \& CLs } \\
\hline & \multicolumn{3}{|c|}{ DI } & \multicolumn{3}{|c|}{ I } & \multicolumn{3}{|c|}{ OW } & \multicolumn{3}{|c|}{ OD } \\
\hline & CLO & CL1 & CL2 & CL0 & CL1 & CL2 & CLO & CL1 & CL2 & CLO & CL1 & CL2 \\
\hline$O p_{1, N}$ & .72 & .13 & .15 & .99 & .005 & .005 & .86 & .09 & .05 & .84 & .08 & .08 \\
\hline $\mathrm{Op}_{2, \mathrm{~N}}$ & .16 & .77 & .07 & .89 & .105 & .005 & .78 & .17 & .05 & .80 & .15 & .05 \\
\hline
\end{tabular}

of DI on signal propagation, which needs to be compensated by $\mathrm{CE}$ techniques. The deviation between outdoor scenarios to $\mathrm{I}$ is instead reduced, with an average increase of $1.43 \mathrm{~dB}$ for $\mathrm{Op}_{1, \mathrm{~N}}$ and $5.82 \mathrm{~dB}$ for $\mathrm{Op}_{2, \mathrm{~N}}$. Comparing the operators, $\mathrm{Op}_{1, \mathrm{~N}}$ consistently provides better NB-IoT coverage $(3.95 \mathrm{~dB}$ on average, and statistically significant for the I scenario), which ties back to the results on deployment statistics and optimality.

Coverage Levels: To better understand how coverage is affected by operators' configurations, we report in Table II the ratio of being in a specific CL, grouped per scenario and split by operator. We retrieve the CL readings by monitoring via TSMA6 the RA attempts performed by the Exelonix module. We then evaluate the ratio as the number of readings for a CL divided by the total number of CL readings. By decoding SIB2 messages, we observe that the operators apply different RSRP thresholds for the $\mathrm{CL}$ estimation, $5 \mathrm{~dB}$ more conservative for $\mathrm{Op}_{2, \mathrm{~N}}$, which is thus more likely to work at higher CLs. When combined with RSRP values of the serving CID, the different thresholds explain the main results in Table II. First, $\mathrm{Op}_{2, \mathrm{~N}}$ has in general lower coverage than $\mathrm{Op}_{1, \mathrm{~N}}$, and thus tries to enhance NB-IoT reliability by operating at higher CLs more, at the cost of higher energy consumption and more repetitions. This is evident in DI, where $\mathrm{Op}_{2, \mathrm{~N}}$ works predominantly in CL1 while $\mathrm{Op}_{1, \mathrm{~N}}$ exhibits a high ratio of 


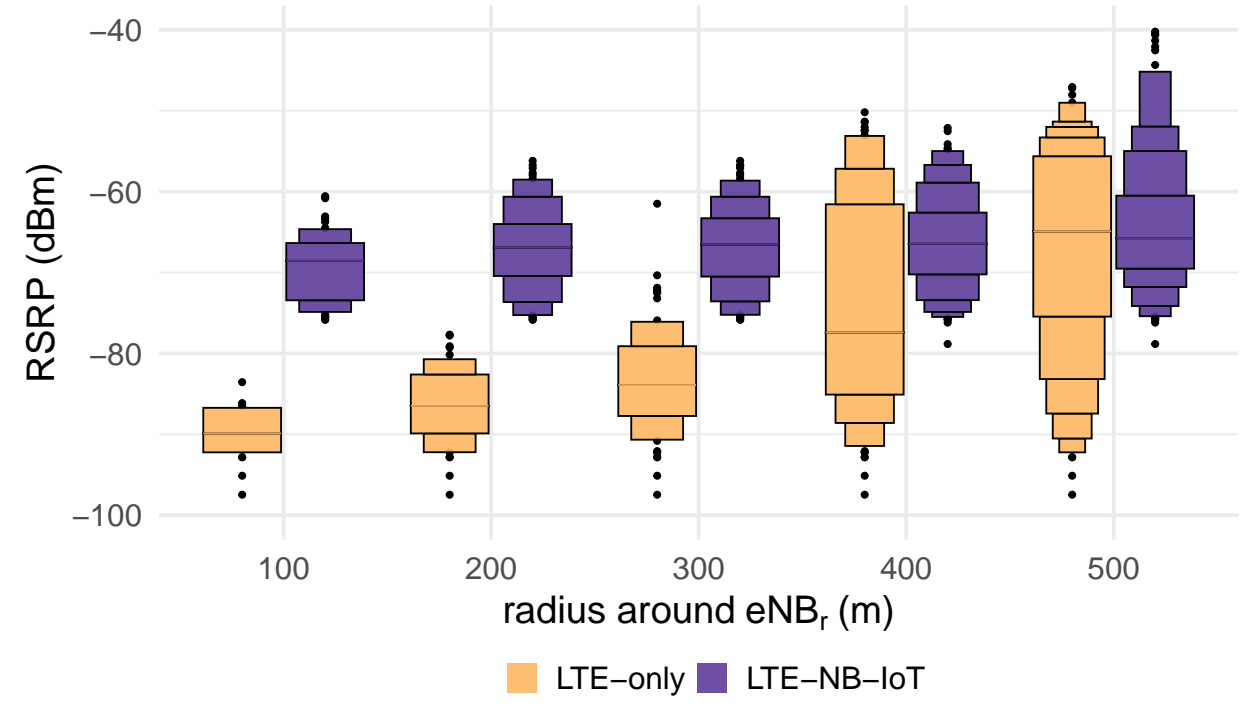

Fig. 3: Network deployment performance evaluation in terms of RSRP [dBm] for the in-band Op ${ }_{1, I}$ deployments.

operating at CLO. Second, operations at CLO are predominant in I, OW, and OD scenarios, showing that, in generic urban environments, NB-IoT is likely to work at radio conditions similar to LTE. Finally, we observe that outdoor scenarios increase the ratio of being at higher CLs when compared to the indoor case, even though higher average RSRP is observed for the former (Figure 2). We argue that this effect is due to the higher heterogeneity of the outdoor scenarios, in terms of radio and mobility aspects. The result suggests that in more heterogeneous and dynamic scenarios, the current CL estimate procedure may be sub-optimal and cause several attempts before the RA succeeds, leading to energy inefficiency.

Takeaways: NB-IoT results in significant coverage improvements with respect to LTE, but operators' configurations have a direct impact on how devices perceive the coverage, execute their operations, and perform in terms of QoS. Empirical data can be used to refine the models adopted in previous work [8], resulting in better modeling NB-IoT coverage and propagation which can pave the way to optimize deployments, configurations, and QoS.

\section{In-band and Guard-band deployment}

In this subsection, we perform a comparison between inband and guard-band modes, quantifying their impact on coverage. As mentioned before, the in-band mode may challenge NB-IoT/LTE coexistence in case of partial NB-IoT deployment across the LTE infrastructure.

We hence focus on in-band mode, and look into the Italian dataset to find a situation of partial NB-IoT deployment for operator $\mathrm{Op}_{1, \mathrm{I}}$. Our goal is to study the characteristics of the NB-IoT signal around an LTE-only eNB, denoted as $\mathrm{eNB}_{\mathrm{r}}$, toward discovering and quantifying potential interference from the LTE signal. Hence, we consider $\mathrm{eNB}_{\mathrm{r}}$ and a NB-IoTenabled eNB, denoted as $\mathrm{eNB}_{\mathrm{x}}$. We then draw two circles around $\mathrm{eNB}_{\mathrm{r}}$ and $\mathrm{eNB}_{\mathrm{x}}$, and isolate all RSRP readings from $\mathrm{eNB}_{\mathrm{x}}$, captured in the intersection of the two. We repeat the process by increasing the radius around $\mathrm{eNB}_{\mathrm{r}}$, and appending new RSRP readings each time. We compare this scenario with a similar topology for $\mathrm{Op}_{1, \mathrm{I}}$, where, however, both $\mathrm{eNB}_{\mathrm{r}}$ and $\mathrm{eNB}_{\mathrm{x}}$ are NB-IoT-enabled. To provide a fair comparison, we select almost symmetric configurations, with analogous distance between the two eNBs. Last, we repeat the same analysis for the guard-band operator $\mathrm{Op}_{2, \mathrm{I}}$.

Figure 3 shows the NB-IoT RSRP distribution as a function of the radius around $\mathrm{eNB}_{\mathrm{r}}$ between 100 and 500 meters (the radius around $\mathrm{eNB}_{\mathrm{x}}$ is fixed to the distance between the two eNBs), grouped by scenario, i.e., LTE-only vs. LTE-NB-IoT $\mathrm{eNB}_{\mathrm{r}}$, for an in-band deployment of $\mathrm{Op}_{1, \mathrm{I}}$. We observe that the effect of interference from LTE is visible especially in close proximity to $\mathrm{eNB}_{\mathrm{r}}$, while it diminishes as the radius increases and finally vanishes at around 500 meters. Contrarily, we observe no interference impact at different radii for the guard-band deployment of $\mathrm{Op}_{2, \mathrm{I}}$, confirming that there is no visible interference for the guard-band deployment. We observe similar trends for the guard-band deployment of $\mathrm{Op}_{3, \mathrm{I}}$.

In the supplementary material [9], we show the analyzed topology, present guard-band results and also validated the statistical significance of the results.

Takeaways: The in-band mode poses coexistence challenges that need to be carefully considered. The empirical assessment of interference is key for the derivation of improved mitigation schemes, also in light of near future transition to $5 \mathrm{G}$, which leads to further coexistence challenges [13].

\section{CONClusion}

In this paper, we present the first publicly available measurement campaign and analysis of NB-IoT on operational networks, focusing on aspects related to network deployment strategies and coverage. By leveraging the collected dataset, we first focus on network deployment, highlighting that empirical studies can pinpoint sub-optimal deployments and pave the way for better deployments with decreased costs and 
energy consumption. We then show that a dense reuse of the LTE RAN for deploying NB-IoT results in a significant coverage increase with respect to LTE, across heterogeneous scenarios and environments. We also analyze how operatorspecific configurations affect end-devices' operations, leading to different estimates of the coverage quality. Finally, we assess the impact of in-band and guard-band modes in terms of LTE interference, showing a non-negligible negative effect of the former, and pinpointing how empirical measurements can help to assess interference and derive mitigation schemes. The open-source nature of our dataset and visualization platform enables further data exploration toward the discovery of new insights and research perspectives.

\section{ACKNOWLEDGMENT}

This work is partially funded by the EU H2020 5GENESIS (815178), and by the Norwegian Research Council project MEMBRANE (250679).

\section{REFERENCES}

[1] H. S. Dhillon, H. Huang, and H. Viswanathan, "Wide-area wireless communication challenges for the internet of things," IEEE Commun. Mag., vol. 55, no. 2, pp. 168-174, 2017.

[2] O. Liberg et al., Cellular Internet of Things: From Massive Deployments to Critical 5G Applications, 2nd Edition. Academic Press, 2020.

[3] U. Raza, P. Kulkarni, and M. Sooriyabandara, "Low power wide area networks: An overview," IEEE Commun. Surveys Tuts., vol. 19, no. 2, pp. 855-873, 2017.

[4] Y.-P. E. Wang et al., "A primer on 3gpp narrowband internet of things," IEEE Commun. Mag., vol. 55, no. 3, pp. 117-123, 2017.

[5] W. Yang et al., "Narrowband wireless access for low-power massive internet of things: A bandwidth perspective," IEEE Wireless Commun., vol. 24, no. 3, pp. 138-145, 2017.

[6] M. Chen et al., "Narrow band internet of things," IEEE Access, vol. 5, pp. 20557-20577, 2017.

[7] "Nb-iot deployment guide to basic feature set requirements," https://www.gsma.com/iot/wp-content/uploads/2019/07/ 201906-GSMA-NB-IoT-Deployment-Guide-v3.pdf， accessed: Feb. 2020.

[8] M. Lauridsen et al., "Coverage comparison of gprs, nb-iot, lora, and sigfox in a $7800 \mathrm{~km}^{2}$ area," in 2017 IEEE 85th Vehicular Technology Conference (VTC Spring). IEEE, 2017, pp. 1-5.

[9] https://mosaic-simulamet.com/nbiotcoverage, accessed: Feb. 2020.

[10] A. Hoglund et al., "Overview of 3gpp release 14 enhanced nb-iot," IEEE Network, vol. 31, no. 6, pp. 16-22, 2017.

[11] "Lte; evolved universal terrestrial radio access (e-utra); requirements for support of radio resource management," https://www.etsi.org/deliver/ etsi_ts/136100_136199/136133/13.03.00_60/ts_136133v130300p.pdf, accessed: Feb. 2020.

[12] B. Martinez et al., "Exploring the performance boundaries of nb-iot," IEEE Internet of Things Journal, vol. 6, no. 3, pp. 5702-5712, 2019.

[13] S. Popli, R. K. Jha, and S. Jain, "A survey on energy efficient narrowband internet of things (nbiot): Architecture, application and challenges," IEEE Access, vol. 7, pp. 16739-16776, 2018.

[14] Y.-C. Wang and C.-A. Chuang, "Efficient enb deployment strategy for heterogeneous cells in 4g lte systems," Computer Networks, vol. 79, pp. 297-312, 2015.

[15] "Lte; evolved universal terrestrial radio access (e-utra); base station (bs) radio transmission and reception," https://www.etsi.org/deliver/ etsi_ts/136100_136199/136104/14.03.00_60/ts_136104v140300p.pdf, accessed: Feb. 2020.

\section{BIOGRAPHIES}

Konstantinos Kousias [S] is a PhD Student at the Faculty of Mathematics and Natural Sciences in University of Oslo affiliated with Simula Research Laboratory. His research focuses on the empirical modeling and evaluation of mobile networks using data analytics and artificial intelligence.

Giuseppe Caso [M] is a Postdoctoral Fellow with the Mobile Systems and Analytics Department at Simula Metropolitan. He received the $\mathrm{PhD}$ degree from Sapienza University of Rome in 2016. His research interests include cognitive communications, distributed learning, and IoT technologies.

Özgü Alay [M] is an Associate Professor with the University of Oslo, Norway. She is also a Chief Research Scientist at Simula Metropolitan. Her research team focuses on the empirical characterization of mobile networks, and the design of protocols and applications for future mobile networks.

Anna Brunstrom [M] is a Full Professor and Research Manager for the Distributed Systems and Communications Research Group at Karlstad University, Sweden. Her research interests include Internet architectures and protocols, techniques for low latency Internet communication, multi-path communication and performance evaluation of mobile broadband systems including 5G.

Luca De Nardis [M] (Ph.D. 2005) is an Assistant Professor in the Department of Information Engineering, Electronics and Telecommunications at Sapienza University of Rome. His research interests focus on indoor positioning, UWB communications, cognitive radio, and routing protocols.

Maria-Gabriella Di Benedetto [F] (Ph.D. 1987) is a Full Professor at the Department of Information Engineering, Electronics and Telecommunications at Sapienza University of Rome. Her research interests include wireless communication systems, in particular impulse radio communications, and speech.

Marco Neri joined Rohde\&Schwarz in 2017 as Application Engineer for Mobile Network Testing. His focus is on $5 \mathrm{G}$ and cellular IoT testing activities worldwide. 